Revue européenne des migrations internationales

vol. 22 - $n^{\circ} 3 \mid 2006$

Turquie 2006 : aux portes de l'Union européenne?

\title{
Élise MASSICARD, L'Autre Turquie. Le mouvement aléviste et ses territoires
}

Stéphane de Tapia

\section{(2) OpenEdition}

1 Journals

Édition électronique

URL : https://journals.openedition.org/remi/4117

DOI : $10.4000 /$ remi.4117

ISSN : 1777-5418

Éditeur

Université de Poitiers

Édition imprimée

Date de publication : 31 décembre 2006

Pagination : 261-262

ISBN : 978-2-911627-43-9

ISSN : 0765-0752

Référence électronique

Stéphane de Tapia, "Élise MASSICARD, L'Autre Turquie. Le mouvement aléviste et ses territoires », Revue européenne des migrations internationales [En ligne], vol. 22 - $n^{\circ} 3$ | 2006, mis en ligne le 27 novembre

2008, consulté le 14 avril 2022. URL : http://journals.openedition.org/remi/4117 ; DOI : https://doi.org/ 10.4000/remi.4117

Ce document a été généré automatiquement le 14 avril 2022.

(c) Université de Poitiers 


\title{
Élise MASSICARD, L'Autre Turquie. Le mouvement aléviste et ses territoires
}

\author{
Stéphane de Tapia
}

\section{RÉFÉRENCE}

Élise MASSICARD, L'Autre Turquie. Le mouvement aléviste et ses territoires, Paris, PUF (Proche Orient), 2005, 361 p., ISBN : 2-12-054947-0

1 L'ouvrage d'Élise MASSICARD, récemment recrutée au CNRS, est issu d'une thèse de doctorat de Sciences Politiques. L'auteure a travaillé en France, en Allemagne, en Turquie, à la définition d'une territorialité transfrontalière, pour ne pas dire transnationale (elle-même préférant employer le terme «trans-étatique »), centrée sur l'appartenance à une confession originale, généralement peu connue hors de Turquie, l'alévisme. L'alévisme et le bektachisme qui lui est historiquement et généalogiquement lié sont deux mouvements religieux proches qui ne se confondent toutefois pas entièrement, intimement ancrés dans les différentes manifestations de la culture turcomusulmane, qu'il s'agisse des Balkans ou de l'Anatolie. Bien que le terme dérive du nom d'Ali, neveu et gendre du Prophète, comme l'arabe alawi (alaouite, ce terme pouvant tout aussi bien désigner la minorité au pouvoir en Syrie que la dynastie chérifienne au Maroc, dans des circonstances historiques complètement différentes, l'un désignant, comme en Turquie une confession hétérodoxe issue du chiisme, l'autre une dynastie marocaine garante du Sunnisme orthodoxe !) et soit issu historiquement de la partition entre musulmans orthodoxes et chiites, très tôt dans l'histoire de l'islam, il est difficile de réduire l'alévisme comme le bektachisme à un simple courant d'obédience chiite. Ce même alors que l'Ali des Alévis ou des Bektachis turcs ressemble trait pour trait à celui des images pieuses fréquentes en Iran, Pakistan, Afghanistan, Liban... chiites. Ali ressemble d'ailleurs très fortement au Jésus des images chrétiennes, double blasphème pour des Sunnites majoritaires qui rejettent toutes les représentations humaines. De nombreux débats passionnés en Turquie et en émigration, comme parmi les spécialistes européens, posent la question du caractère véritable de l'alévisme, philosophie, religion 
autonome ou simple hétérodoxie musulmane. Cela ne me semble pas l'essentiel dans l'apport d'Élise Massicard (qui d'ailleurs présente plusieurs courants actuels de l'alévisme qui se posent justement la question de l'appartenance à l'islam en général et au chiisme en particulier) qui a fait un travail de politologue et non d'ethnologue ou d'anthropologue et qui, par ailleurs, souligne ces débats internes à l'alévisme qui se pose effectivement la question de la définition du mouvement, religion, philosophie ou mode de vie. Le sous-titre intéressera les géographes, car il est ici question de territoires et de territoires multiples liés entre eux par des mouvements migratoires. $\mathrm{Au}$ départ, le territoire d'origine, pour le cas présent, en grande partie tribal et kurde (région de Sivas, en Anatolie centrale), en positions intermédiaires, les agglomérations turques, zones de réception de l'exode rural récent, comme Ankara ou Istanbul, à l'arrivée les cités industrielles européennes, lieux d'une immigration turque nombreuse et au retour (ici surtout Berlin, agglomération comprenant de nombreux Alévis parmi la population immigrée originaire de Turquie), éventuellement, les espaces politiques turcs où se réinsèrent des individus qui font la navette entre tous ces territoires.

2 L'ouvrage est organisé en trois parties et onze chapitres, suivant le long prologue cité qui est véritablement utile pour la suite de la lecture. Ces trois chapitres sont intitulés :

- Un mouvement polymorphe et divisé.

- Le difficile ancrage du mouvement en Turquie.

- La localisation de l'identité.

3 Ce livre d'Élise Massicard, ou d'ailleurs plusieurs de ses articles (Études du CERI, European Journal of Turkish Studies), offrent une merveilleuse illustration de ce que peut être un "espace transnational » ou plutôt, selon l'auteure, d'un espace qu'il vaudrait mieux qualifier de trans-étatique. La comparaison entre la situation turque, où émergent de très nombreux mouvements tactiquement et idéologiquement opposés, mais malgré tout en recomposition incessante, confrontés aux aléas de la vie politique turque comme à certaines interférences externes (pression européenne sur la démocratisation de la vie politique turque, intérêt de l'Iran chiite, tentatives de "fédérationisme " kurde...), et situation européenne, principalement allemande, où les Alévis jouissent d'une bien plus grande liberté de manœuvre, a amené des évolutions contraires : un relatif émiettement de la revendication alévie, sous diverses pressions qui elles-mêmes sont en constante évolution ; une réelle structuration en Allemagne et au-delà sur tout le champ migratoire turc en Europe. À première vue, l'ouvrage peut paraître éloigné des préoccupations des lecteurs de la REMI : la situation européenne ne fait l'objet que d'un seul chapitre à la fin du livre: "L'Alévisme en Europe: une cristallisation par l'extérieur?» (pp. 279-314). En réalité, tout le texte est traversé, ce qui n'est certes pas un accident, par des considérations entre ceux que l'on pourrait désigner comme « les Alévis d'ici " et "les Alévis de là-bas », ainsi les pp. 269-273 avec les paragraphes consacrés à «Berlin : l'alévisme en migration ». L'auteure explique clairement que les enjeux ne sont pas les mêmes en Europe occidentale et en Turquie, mais les acteurs sont souvent en relation constante et il n'est pas rare que les Alévis d'Europe cherchent à influer sur la politique turque envers leurs collègues anatoliens comme ceux de Turquie se reposent en partie sur ceux d'Europe pour défendre leurs droits en Turquie. Nous citerons parmi ces acteurs de premier plan plusieurs députés du Bundestag (Cem Özdemir), Ozan Ceyhun, de Länder de Berlin (Ozan Ceyhun, Özcan Mutlu) ou du Parlement européen (Ali R|za Gülçiçek, à la fois député turc de la gauche social- 
démocrate, député européen et ancien dirigeant de la plus puissante fédération associative d'Allemagne : l'AABF ou Almanya Alevi Birlkileri Federasyonu).

4 L'étude d'Élise Massicard, qui a donné en parallèle plusieurs articles, dont l'un, publié dans les Études $d u$ CERI, me semble être un modèle du genre pour qui s'intéresse à la définition des réseaux migrants transnationaux, et d'une grande finesse d'analyse (voir par exemple le sous-chapitre intitulé «les territoires du mouvement aléviste » dans l'introduction, pp. 12-15). Elle ne s'appesantit pas sur les problèmes de définition de l'essence de l'alévisme, ce n'est pas son objet, mais elle décrit tous les courants de pensée, qui mobilisent ethnologie, théologie, politologie, sociologie..., agitant la population alévie, tant en Turquie qu'en émigration, la place et le rôle insignes de la jeunesse alévie en mal de reconnaissance et approfondit toutes les pistes de recherche qui font de l'alévisme turc, qu'il soit d'ailleurs turc ou kurde, un passionnant mais extrêmement complexe champ d'étude sur la demande de démocratisation de la population turque dans son ensemble. Les circonstances historiques de la naissance, du développement, de l'évolution de l'alévisme sont brossés à grands traits, mais cette analyse ne me semble en aucun cas trahir l'historicité du mouvement dans sa complexité (Prologue pp. 17-51: Des « trajectoires historiques complexes»).

Cet ouvrage permet une lecture nouvelle et facilitée d'un fait social global complexe, qui intéresse autant la politique intérieure turque que celles de chacun des pays européens d'immigration. L'alévisme apparaît en pleine et rapide transformation, due tant au fait de la nouveauté du fait migratoire international pour la population rurale turque qu'à un réel mouvement de démocratisation de la société turque. Il y a sans doute encore beaucoup à écrire sur l'alévisme en Turquie et dans le champ migratoire, mais l'ouvrage d'élise Massicard est sans nul doute une étape incontournable pour les spécialistes de la Turquie contemporaine et un excellent exemple d'étude d'une population transnationale, ou pour reprendre le terme de l'auteure, trans-étatique.

\section{AUTEURS}

\section{STÉPHANE DE TAPIA}

CNRS - UMR 7043 ; Cultures \& Sociétés en Europe/Strasbourg, associé à l'UMR 6588, MIGRINTER/ Poitiers. 